\title{
The Inhibitory Post-synaptic Potential in Intestinal Smooth Muscle Investigated with Intracellular Microelectrode
}

\author{
By \\ Taizo Suzuki and Hachiro Inomata \\ Department of Applied Physiology, Tohoku University School of \\ Medicine, Sendai
}

(Received for publication, November 28, 1963)

\begin{abstract}
By selective stimulation of intramural sympathetic nerve fibers, the inhibitory post-synaptic potential in intestinal smooth muscle cell could be recorded intracellularly. This inhibitory potential, with the exception of direction, was found to be similar, in many of its properties, to the excitatory post-synaptic potential in vas deferens.
\end{abstract}

Recently, numerous investigations have been performed on the electrical activity of the smooth muscle cells from various sources and it is now generally accepted that the smooth muscle cells generate the action potential, similar to those of the other excitable tissues. However, there have not been sufficient electrophysiological data on the transmission processes of excitation and inhibition from autonomic nerves to the smooth muscle cells ${ }^{3}$ ). Concerning the excitatory transmission process, Burnstock and Holman ${ }^{1,2}$ ) have demonstrated the experiments that the stimulation of the hypogastric nerve gave rise to slow depolarization on the muscle cell of vas deferens, which they named the junction potential. They showed that the repetitive stimulations brought about the summation of junction potentials, giving rise ultimately to the initiation of the action potential.

In the experiment to be described here, the inhibitory post-synaptic potential in intestinal smooth muscle cell has been recorded with an intracellular microelectrode. In addition, information has been obtained about the effect of changes in intensity and frequency of stimulation on this potential.

\section{METHODS}

Experimental preparation

All the experiments were carried out with the isolated preparations from guinea pig taenia coli. Taenia coli was removed by cutting out pieces and each

鈴木泰三，猪欢八郎 
piece was mounted in a plastic chamber, keeping its length in situ. The Krebs's solution used in experiments contained $(\mathrm{mM}): \mathrm{NaCl} 155, \mathrm{KCl} 3.8, \mathrm{CaCl}_{2} 1.8, \mathrm{MgCl}_{2}$ 0.2 , glucose and phosphate buffer. The solution, when aerated, had a $\mathrm{pH}$ of 7.3 7.4 and its temperature could be maintained at any level.

\section{Stimulating}

Electrical stimulation of rectangular pulse current was applied by means of massive silver electrodes through isolation unit. The strength of stimulating current was measured as the voltage drop across the resistor which was included in the stimulating circuit. The voltage drop was displayed on one beam of oscilloscope. The intensity and duration of stimulation was adjusted properly to be able to stimulate the intramural nerve element selectively, since the threshold value for nerve element is lower than for muscle cell and its difference is greater with shorter stimulation ${ }^{4-6)}$. After the muscle was kept in Krebs's solution for a long time, however, the threshold voltage for nerve element became higher than for muscle cells.

\section{Recording}

Intracellular potentials were recorded by the glass microelectrode method. The electrodes were filled with $3 \mathrm{M}-\mathrm{KCl}$ and had a high resistance, optimally between 40 to $70 \mathrm{M} \Omega$. The sign from the electrode was led to a cathode-follower of conventional design and the output of this, through DC amplifier, was displayed on another beam of oscilloscope.

\section{RESULTS}

\section{General properties of the inhibitory post-synaptic potential}

The inhibitory synpatic action on the smooth muscle cell membrane is associated with a hyperpolarization. Typical record could be seen in Fig. 1. Such a hyperpolarizing potential is the inhibitory post-synaptic potential (IPSP) in smooth muscle. Hyperpolarization is first observed about 50 to $70 \mathrm{msec}$ after the stimulation; thereafter it reaches to a summit in further 80 to $120 \mathrm{msec}$ and then decays to the resting level slowly. This long latency is not affected by the strength of stimulation. But the amplitude of potential change is generally graded, increasing with the intensity of the applied stimulation. Thus, if weak stimuli are applied at low frequency, amplitude of potential change is small, so that decay of potential is complete before the next hyperpolarization begins; the spontaneous discharge is not suppressed (top in Fig. 1). As to be expected, the marked inhibition is obtained by increasing the intensity or frequency of the stimulation (middle and bottom in Fig. 1).

Interaction of the inhibitory post-synaptic potential and spike potential

As the next step, the effect of setting up the inhibitory post-synaptic potential during the refractory period after the spike potential was examined, in 


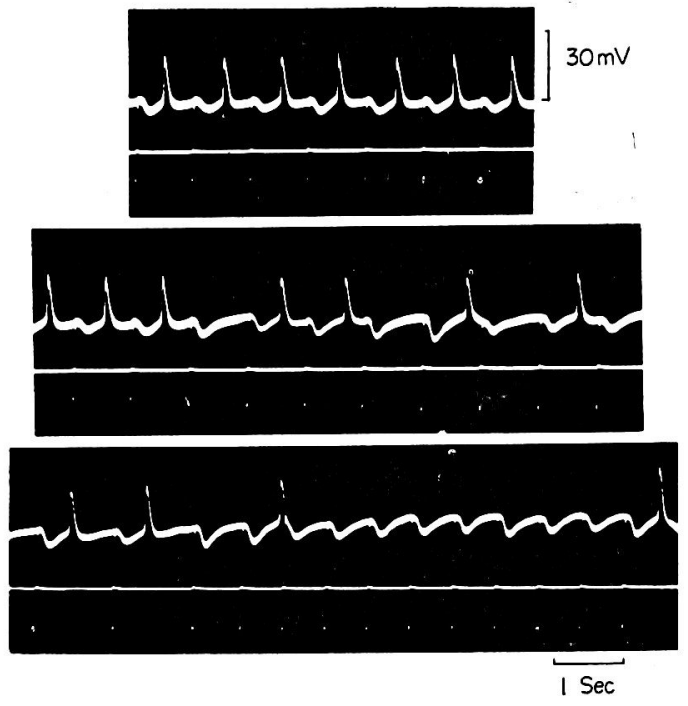

Eig. 1. Inhibitory post-synaptic potential in taenia coli of guinea pig recorded with intracellular microelectrode. Top, response to weak stimulation. Each stimulus gives rise to a slow hyperpolarization (inhibitory post-synaptic potential), which is insufficient to suppress the spontaneous discharge. Middle, inhibitory response (which is signed by dropping of spontaneous discharge) obtained by increasing the stimulus strength. Bottom, marked inhibition caused by repetitive stimulation at higher frequencies. The lower trace in each record indicates the strength of stimulation.
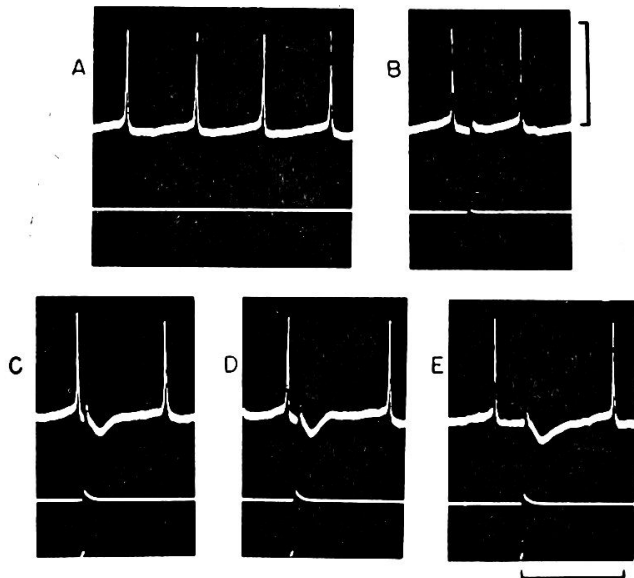

Fig. 2. Intracellular post-synaptic potential in taenia coli of guinea pig. A, control (spontaneous spike discharge); B, subthreshold stimulation ; $\mathrm{C}$ to $\mathbf{E}$, inhibitory post-synaptic potential evoked by stimulation applied at the various intervals after spike potential. The lower trace in B to E indicates the strength of stimulation. Time marker, 1 second ; voltage calibration, $30 \mathrm{mV}$. 
order to find out the interaction of the spike potential and the inhibitory post-synaptic potential. The stimuli were applied at the various intervals after the spike potential. The result was illustrated in Fig. 2. These inhibitory postsynaptic potentials (shown in Fig. 2, C to E) were closely similar each other in size, time course and latency. This result indicates that the generation of the inhibitory post-synaptic potential is not affected by the refractoriness.

\section{DISCUSSION}

Our records reveal that latency and time course of the inhibitory postsynaptic potential in taenia coli, with exception of direction, is closely similar to that of the excitatory post-synaptic potential in vas deferens. This similarity is interpreted as due to the fact that both the inhibitory nerve fibers in taenia coli and the excitatory nerve fibers in vas deferens are equally sympathetic nerve fibers and the transmitter involved is noradrenaline.

Recently, Burnstock ${ }^{7)}$ has demonstrated the inhibitory post-synatpic potential in smooth muscle recorded with sucrose-gap method. This method is excellent in smooth muscle research ${ }^{8}$. But a weakness of this method is that the height of the recorded potential would be reduced by temporal dispersion of the individual spikes and the occurrence of inactive cells. However, it was confirmed by our experiments that the latency and time course of the inhibitory postsynaptic potential recorded with the sucrose-gap method were generally similar to our record obtained with an intracellular microelectrode.

Burnstock and Holman ${ }^{2}$ called the depolarization of vas deferens in response to nerve stimulation, a junction potential. We feel that the term junction potential would be reserved for the potential between adjacent muscle cell ${ }^{10)}$. It seems more likely that, in smooth muscle, depolarization or hyperpolarization in response to each stimulus would be termed excitatory or inhibitory post-synaptic potential in smooth muscle, since this potential is virtually identical, in its properties, to those observed in the central nervous system ${ }^{9}$.

This work was supported in part by the Grant of the Population Council.

\section{References}

1) Burnstock, G. \& Holman, M.E., Nature, 1960, 187, 951.

2) Burnstock, G. \& Holman, M.E., J. Physiol. (Lond.), 1961, 155, 115.

3) Gillespie, J.S., J. Physiol. (Lond.), 1962, 162, 54.

4) Whalen, W.J., Fishman, N. \& Erickson, R., Amer. J. Physiol., 1958, 194, 573.

5) Amory, W.D. \& West, T.C., J. Pharmacol. exp. Ther., 1962, 137, 14.

6) Lewartowski, B., Nature, 1963, 199, 76.

7) Burnstock, G., lecture at the Tokyo University, September, 1963.

8) Burnstock, G. \& Straub, R.W., J. Physiol. (Lond.) 1958, 140, 156.

9) Coombs, J.S., Eccles, J.C. \& Fatt, P., J. Physiol. (Lond.) 1955, 130, 326.

10) Goto, M., Kuriyama, H. \& Abe, Y., Proc. imp. Acad. Japan, 1960, 36, 509.

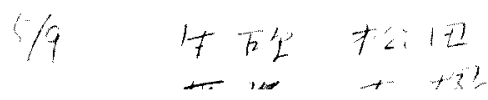

